\title{
The pre-ligand binding assembly domain: a potential target of inhibition of tumour necrosis factor receptor function
}

\author{
Francis Ka-Ming Chan
}

Building 10, Room Immunology, National Institute of Allergy and Infectious Diseases, National Institutes of Health, Bethesda, MD 20892-1892, USA

Correspondence to: DrChan (fchan@nih.gov) 11N311, Laboratory of

\begin{abstract}
Signalling by the tumour necrosis factor receptors (TNFR) is thought to be mediated by the binding of the trimeric ligand TNF to three monomeric subunits of the receptor. This ligand induced trimerisation model of TNFR signalling is mainly supported by crystallographic data of the p60 TNFR-1 and TNF $\beta$ complex in which the trimeric ligand interdigitates between the individual receptor chains and prevents the receptor subunits from interacting with each other. Recently, a domain $\mathrm{NH}_{2}-$ terminal to the ligand binding domain in the extracellular region of p60 TNFR-1, p80 TNFR-2 and Fas was identified that mediates receptor self association before ligand binding. This pre-ligand binding assembly domain or PLAD is critical for assembly of functional receptor complexes on the cell surface and may provide a potential target in the design of future novel therapeutics against diseases mediated by members of the TNFR family of receptors.

(Ann Rheum Dis 2000;59(suppl I):i50-i53)
\end{abstract}
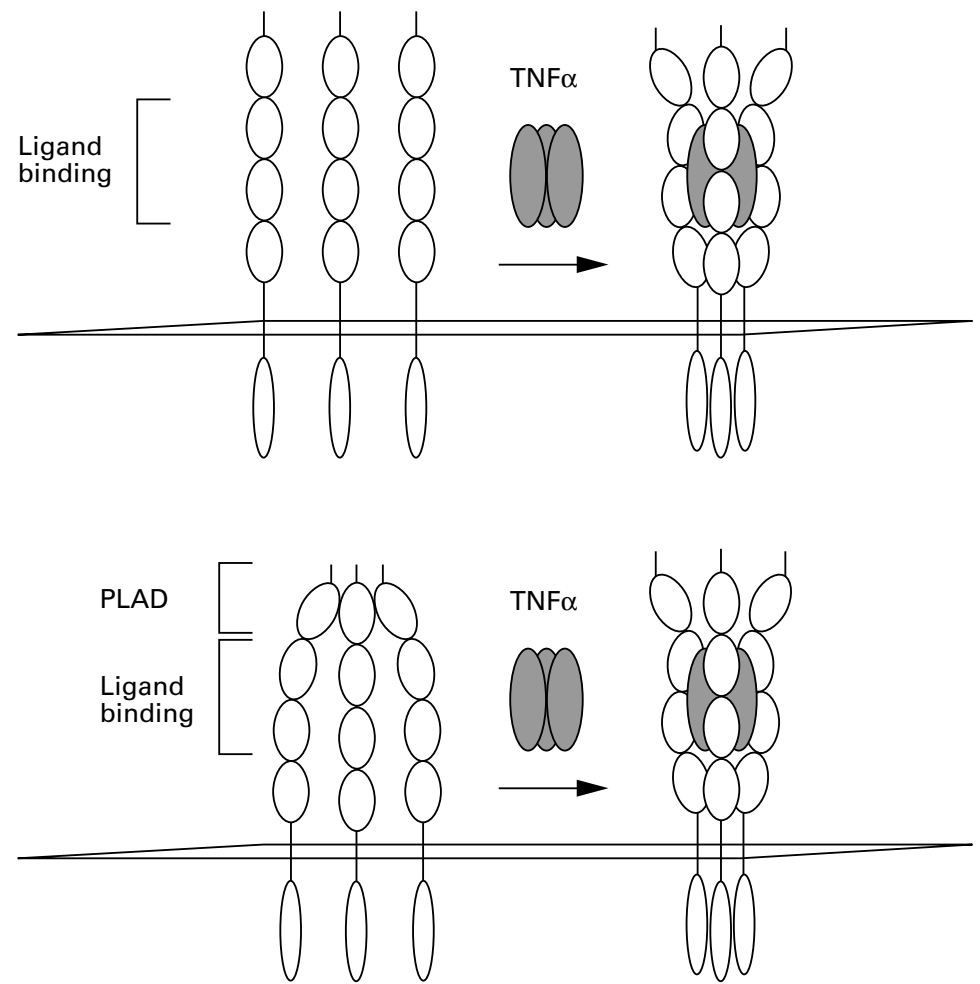

Figure 1 The two models of TNFR signalling. The ligand induced trimerisation model (top) and the pre-ligand assembly model (bottom). The $\mathrm{NH}_{2}$-terminal PLAD domain and the ligand binding domain are shown.
Tumour necrosis factor (TNF) is a cytokine that plays an important regulatory part in both healthy and diseased immune responses. ${ }^{12}$ $\mathrm{TNF} \alpha$ signals through two distinct receptors, the $60 \mathrm{kDa}$ TNF receptor 1 (TNFR-1 or p60) and the $80 \mathrm{kDa}$ TNFR-2 or p80. The TNFRs represent a growing family of cell surface receptors including Fas/CD-95/Apo-1 and CD40, etc, which regulate various aspects of the immune system. ${ }^{3}$ Failure of proper signalling of these receptors often leads to pathological states within the immune system. For example, hereditary heterozygous mutations in the Fas receptor are the most prevalent cause of paediatric patients suffering from autoimmune lymphoproliferative syndrome (ALPS), a disease that is characterised by lymphoproliferation and defective apoptosis induction. ${ }^{45}$ The mutant Fas receptors were thought to dominantly inhibit the function of the wild type receptor through ligand dependent recruitment of the wild type and mutant receptor chains, thus forming a defective, non-signalling receptor complex.

The ligand induced trimerisation model As mentioned above, the conventional model of TNFR signalling proposes that the trimeric ligand TNF recruits three separate chains of the receptor through ligand induced trimerisation. ${ }^{6}$ The resulting complex would then allow juxtaposition of the cytoplasmic domains to facilitate recruitment of downstream signalling components (fig 1). ${ }^{7}$ Evidence supporting this model of TNFR signal transduction comes from both solution studies ${ }^{8}$ and crystallography. ${ }^{9}$ In both cases, a three to three ratio of ligand to receptor complex was observed. The ligand bound TNFR-1 structure ${ }^{9}$ and the more recently described Trail receptor structure ${ }^{1011}$ both reveal a similar architecture in which the trimeric ligand interdigitates between the three receptor chains. The individual receptor chains in the complex are thus prevented from making contact with each other. The crystallographic data were corroborated by transfection studies where a cytoplasmic domain truncated human TNFR-1 was introduced into a mouse fibroblast cell line. The truncated human receptor was able to dominantly interfere with apoptosis induced by human $\mathrm{TNF} \alpha$, which binds to both the human and mouse TNFR-1, but not apoptosis induced by antibody to the mouse TNFR-1. ${ }^{12}$ These results and others are consistent with a ligand induced receptor trimerisation model of signal initiation. 
Table 1 TNF-like ligands with multiple receptors. The alternative names of the ligands and receptors are listed in parentheses

\begin{tabular}{ll}
\hline Ligand & Receptor \\
\hline TNF $\alpha$ & TNFR-1 (p55, p60) \\
& TNFR-2 (p75, p80) \\
TNF $\beta(\mathrm{LT} \alpha)$ & TNFR-1 (p55, p60) \\
LT $2 \beta 1$ & TNFR-2 (p75, p80) \\
& TNFR-1 (p55, p60) \\
TRAIL (Apo2L) & TNFR-2 (p75, p80) \\
& DR4 (TRAIL-R1) \\
& DR5 (TRAIL-R2, TRICK2, Killer, Apo2) \\
& DcR1 (TRAIL-R3, TRID) \\
& DcR2 (TRAIL-R4, TRUNDD, LIT) \\
RANKL (TRANCE, OPGL, ODF) & OPG (FDCR-1, OCIF) \\
& RANK (TRANCE-R) \\
LIGHT & OPG (FDCR-1, OCIF) \\
& HVEM (HveA) \\
& LT $\beta$ R
\end{tabular}

\section{The pre-ligand assembly model}

Recent evidence, however, suggests that an alternative model of TNFR signalling should be considered. According to the ligand induced trimerisation model, addition of TNF to a cell that expresses both p60 and p80 TNFRs would theoretically lead to the formation of mixed trimer complexes. Such mixed complexes would be expected to be non-signalling as productive recruitment of downstream signalling proteins requires the formation of a homotrimeric cytoplasmic interface (the death domain for p60 and the TRAF binding domain for $\mathrm{p} 80$ ). However, in contrast with the prediction of the model, p 80 TNFR-2 can increase the cytotoxicity of p60 TNFR-1, especially when over-expressed in cell lines. ${ }^{13-17}$ In fact, the potential to form mixed trimers is not restricted to p60 and p80 TNFR. Many of the TNF family of ligands, including the TNF related apoptosis inducing ligand (TRAIL), can bind to multiple receptor partners (table 1). ${ }^{18-26}$ As these receptors are responsible for transduction of distinct signals, the formation of mixed receptor trimers can have deleterious effects on the cellular response to external stimuli. The promiscuous nature of binding specificity within the TNFR superfamily thus challenges the validity of the ligand induced trimersiation model.

Another line of evidence that points to the deficiency of the ligand induced trimerisation model comes from the ALPS patients who were mentioned earlier. While most of the ALPS type 1 patients (patients with mutations in the Fas or Fas-L genes) harbour mutations in the cytoplasmic death domain of the receptor, a number of these patients were found to possess heterozygous mutations in the extracellular domain of the receptors. ${ }^{27-29}$ Some of these extracellular domain mutations result in nonligand binding receptors. As these mutant receptors cannot bind Fas-L, they were not expected to enter into functional signalling complexes with the wild type receptors. However, Fas induced apoptosis in the lymphocytes of these ALPS patients was defective. When coexpressed with the wild type receptors, these non-ligand binding Fas receptors can also dominantly interfere with the apoptotic activity of the wild type receptors. ${ }^{30}$ These results suggest that the mutant receptor may somehow be able to associate with the wild type receptor in the absence of ligand, in contradiction with the predictions of the ligand induced trimerisation model.

\section{The identification of the PLAD}

The aforementioned evidence thus raised the question of whether TNFR family receptors can associate with each other prior to ligand binding. Although the death domain and the TRAF binding domain in the cytoplasmic region of TNFR family receptors are known to be able to mediate trimerisation, this event is only associated with signal transduction upon ligand binding or over-expression of the receptor. $^{31}$ Thus, the putative pre-ligand assembly domain is likely to reside outside of the cytoplasmic domain of the receptor. One outstanding feature shared among TNFR-like receptors is the tandem repeats of cysteine rich domains (CRDs) in the extracellular domain that is homologous among members of the family. The ligand binding domains for p60/ p80 TNFRs and Fas are made up of the second and third CRDs. Although the first CRD for TNFRs is also highly conserved and has been shown to be critical for ligand binding, the mechanism by which it contributes to receptor function was unclear. ${ }^{32-35}$ Interestingly, the unliganded p60 receptor was crystallised as dimers. Of particular interest is a structure obtained at neutral, physiological $\mathrm{pH}$ that shows the p60 TNFR-1 extracellular domain as parallel dimers with extensive contacts in the first CRD. ${ }^{36}{ }^{37}$ Using a combination of biochemical and biophysical techniques, the extracellular domains of p60, p80 and Fas were indeed shown to self associate in the absence of ligand. For instance, using spectral variants of the green fluorescent protein (GFP) ${ }^{38}$ fused to p60 TNFR-1, receptor specific interaction between p60-p60 can be detected in living cells using the fluorescence resonance energy transfer (FRET) technique. ${ }^{39} 40$ The energy transfer is homospecific as it cannot be detected between p60 and p80 or Fas. Subsequent mapping experiments confirm the region overlapping the first CRD of p60, p80 and Fas called the PLAD (pre-ligand binding assembly domain) to be necessary and sufficient for mediating receptor association in the absence of ligand, ${ }^{304} 42$ a finding that agrees with the unliganded p60 TNFR-1 crystal structure. For example, replacing the PLAD of p60 with that of $\mathrm{p} 80$ can redirect the association of the chimeric receptor to $\mathrm{p} 80$ but not $\mathrm{p} 60 .{ }^{41}$ Two other TNFR family members, DR4 and CD40, also demonstrate ligand independent self association. ${ }^{41}$ Thus, the PLAD seems to be a mechanism by which the different receptors within the TNFR family sort themselves into distinct homotypic complexes, thus avoiding cross inhibition between receptors.

The discovery of the PLAD also highlights the significance of the pre-associated receptor complex in ligand binding. ${ }^{32-35}$ Removal of the PLAD or mutations within the PLAD that disrupts PLAD mediated self association renders the receptor incapable of binding the ligand. This loss of ligand binding can be rescued by replacing the PLAD deleted receptor with a 
PLAD from a heterologous receptor. ${ }^{41} \mathrm{Al}-$ though receptor pre-ligand association is required for ligand binding, mutations in the CRD2 that abolish ligand binding do not preclude the receptor from self association. These CRD2 mutant receptors can still enter into complexes with the wild type receptors and cause dominant inhibition on the wild type receptor in response to ligand, a result that is reminiscent of the ALPS mutations. ${ }^{41}$ This evidence thus establishes that pre-assembled receptor complexes are the functional, ligand binding form of the receptor (fig 1).

\section{PLAD and human diseases}

What is the importance of the PLAD with regard to human diseases? As mentioned above, ligand independent association of Fas causes dominant inhibition of wild type receptor function in ALPS. A striking feature of type 1 ALPS is that all heterozygous Fas mutations identified so far have retain the PLAD domain. In fact, removal of the PLAD from some of these dominant negative Fas mutants abolished their dominant negative phenotype on the wild type receptor. ${ }^{30}$ Thus, PLAD mediated receptor association contributes to the pathology of ALPS and provides a molecular explanation by which non-ligand binding and signal deficient Fas mutants interfere with wild type receptor function.

Mutation in the extracellular portion of TNFR-1 causes TNFR-1 associated periodic syndromes (TRAPS). ${ }^{43}$ TRAPS is characterised by unexplained episodes of fever and localised inflammation. ${ }^{44}$ Interestingly, the reported mutations are heterozygous, suggesting that they may dominantly interfere with the function of the wild type receptor. In fact, in one of the TRAPS patients, there is reduced shedding of the receptor in response to cellular stimulation. ${ }^{43}$ Although it has not been formally tested, it is tempting to speculate that this effect and other phenotypes related to TRAPS are also caused by dominant interference of the wild type receptor function through PLAD mediated receptor association.

\section{PLAD disrupting agents: potential therapeutic reagents?}

The discovery of the PLAD and PLAD mediated ligand independent receptor assembly provides a novel understanding of the biology of this large family of receptors whose functions are critical to the immune system. An intriguing implication to the discovery of the PLAD is that it may serve as a novel target to the prevention of TNFR signalling in diseases such as rheumatoid arthritis. Currently, target specific treatments of rheumatoid arthritis include blockade of ligand receptor interaction (anti-TNF antibodies) and competition for ligand binding (soluble recombinant receptor proteins). Although the use of these novel treatments in the clinic has yielded promising results, they all carry with them certain disadvantages. For example, the use of anti-TNF antibody may elicit anti-idiotypic responses. Moreover, anti-TNF antibody does not distinguish the interaction of TNF with multiple receptors. For the same reason, soluble recombinant receptor proteins also do not distinguish TNFR-1 from TNFR-2, and may cause undesirable effects by inhibition of receptor function that is not central to disease pathology. On the other hand, the PLAD can be an attractive alternative to designing novel therapeutics against TNFR function. A polypeptide mimicking the PLAD binding contacts can be used to specifically inhibit the assembly of functional receptor complexes. Such polypeptide inhibitors will be receptor specific and "totally self" to the immune system, thus circumventing the danger of eliciting undesirable immune reactions. The receptor specific inhibition of TNFR function may therefore provide an attractive alternative to the current therapeutic approaches. Further work is needed to determine the feasibility and applicability of targeting the PLAD in therapeutic design.

Funding: the author is supported by a Cancer Research Institute/Miriam and Benedict Wolf Fellowship.

Copyright is held by the US Government.

1 Tracey KJ, Cerami A. Tumor necrosis factor, other cytokines and disease. Annu Rev Cell Biol 1993;9:317-43. 2 Tracey KJ, Cerami A. Tumor necrosis factor: a pleiotropic cytokine and therapeutic target. Annu Rev Med 1994;45: 491-503.

3 Lenardo M, Chan FKM, Hornung F, McFarland H, Siegel $\mathrm{R}$, Wang J, et al. Mature T lymphocyte apoptosis-immune $\mathrm{R}$, Wang $\mathrm{J}$, et al. Mature $\mathrm{T}$ lymphocyte apoptosis-immune
regulation in a dynamic and unpredictable antigenic regulation in a dynamic and unpredictable ant

4 Puck JM, Sneller MC. ALPS: an autoimmune human lymphoproliferative syndrome associated with abnormal lymphocyte apoptosis. Semin Immunol 1997;9:77-84

5 Infante A, Britton H, DiNapoliT, et al. The Clinical Spectrum in a large kindred with ALPS due to a Fas mutation that impairs lymphocyte apoptosis. J Pediatr 1998;133: 629-33

6 Smith CA, Farrah T, Goodwin RG. The TNF receptor superfamily of cellular and viral proteins: activation, costimulation, and death. Cell 1994;76:959-66.

7 Wallach D, Varfolomeev E, Malinin N, Goltsev Y, Kovalenko A, Boldin M. Tumor necrosis factor receptor and Fas signaling mechanisms. Annu Rev Immunol 1999; 17:331-67.

8 Loetscher H, Gentz R, Zulauf $\mathrm{M}$, et al. Recombinant $55-\mathrm{kDa}$ tumor necrosis factor (TNF) receptor. Stoichiometry of binding to TNF alpha and TNF beta and inhibition ef TNF activity. J Biol Chem 1991;266:18324-9.

9 Banner D, D'Arcy A, Janes W, et al. Crystal structure of the soluble human $55 \mathrm{kd}$ TNF receptor-human TNF beta complex: implications for TNF receptor activation. Cell 1993;73:431-45.

10 Mongkolsapaya J, Grimes J, Chen N, et al. Structure of the TRAIL-DR5 complex reveals mechanisms conferring specificity in apoptotic initiation. Nat Struct Biol 1999;6: 1048-53.

11 Hymowitz SG, Fuh G, Ultsch M, O'Connell M, Kelley RF, Ashkenazi A, et al. Triggering cell death: The crystal structure of Apo2L/TRAIL in a complex with death receptor 5. Mol Cell 1999;4:563-71.

12 Tartaglia L, Goeddel D. Tumor necrosis factor receptor signaling. A dominant negative mutation suppresses the activation of the $55-\mathrm{kD}$ a tumor necrosis factor receptor. J Biol Chem 1992;267:4304-7.

13 Chan FKM, Lenardo MJ. A crucial role for p80 TNF-R2 in amplifying p60 TNF-R1 apoptosis signals in T lymamplifying p60 TNF-R1 apoptosis sign

14 Heller RA, Song K, Fan N, Chang DJ. The p70 tumor necrosis factor receptor mediates cytotoxicity. Cell 1992;
nat necrosis facto $70: 47-56$.

15 Weiss T, Grell M, Hessabi B, et al. Enhancement of TNF receptor $\mathrm{p} 60$-mediated cytotoxicity by TNF receptor $\mathrm{p} 80$ : requirement of the TNF receptor-associated factor-2 binding site. J Immunol 1997; 158:2398-404

6 Haridas V, Darnay BG, Natarajan K, Heller R, Aggarwal BB. Overexpression of the p80 TNF receptor leads to TNF-dependent apoptosis, nuclear factor-kappa B activation, and c-Jun kinase activation. J Immunol 1998;160: 3152-62

17 Declercq W, Denecker G, Fiers W, Vandenabeele P. Cooperation of both TNF receptors in inducing apoptosis: involvement of the TNF receptor-associated factor binding Cor domain

18 Pan G, Ni J, Wei YF, Yu G, Gentz R, Dixit VM. An antagonist decoy receptor and a death domain-containing nist decoy receptor and a death domain-cont

19 Pan G, O'Rourke K, Chinnaiyan AM, et al. The receptor for the cytotoxic ligand TRAIL. Science 1997;276:111-13. 
20 Schneider P, Thome M, Burns K, et al. TRAIL receptors 1 (DR4) and 2 (DR5) signal FADD-dependent apoptosis :831-6.

21 Screaton GR, Mongkolsapaya J, Xu XN, Cowper AE, McMichael AJ, Bell JI. TRICK2, a new alternatively spliced receptor that transduces the cytotoxic signal from TRAIL. Curr Biol 1997; 7:693-6.

22 Walczak H, Degli-Esposti MA, Johnson RS, et al. TRAILR2: a novel apoptosis-mediating receptor for TRAIL EMBO J 1997; 16:5386-97.

23 Degli-Esposti MA, Smolak PJ, Walczak $\mathrm{H}$, et al. Cloning and characterization of TRAIL-R3, a novel member of the emerging TRAIL receptor family. J Exp Med 1997;186: $1165-70$

24 Degli-Esposti MA, Dougall WC, Smolak PJ, Waugh JY, Smith CA, Goodwin RG. The novel receptor TRAIL-R4 induces NF-kappaB and protects against TRAIL-mediated apoptosis, yet retains an incomplete death domain. Immuapoptosis, yet retains
nity $1997 ; 7: 813-20$.

25 Golstein P. Cell death: TRAIL and its receptors. Curr Biol 1997;7:R750-3.

26 Emery JG, Burke MB, Deen KC, Lyn S, Silverman C, Dul $\mathrm{E}$, et al. Osteoprotegerin is a receptor for the cytotoxic ligand TRAIL.

27 Rieux-Laucat F, Diest FL, Roberts IA, Debatin KM, Fisher A, Villartay JP. Mutations in Fas-associated with human lymphoproliferative syndrome and autoimmunity. Science 1995;268:1347-51.

28 Drappa J, Vaishnaw AK, Sullivan KE, Chu JL, Elkon KB. Fas gene mutations in the Canale-Smith syndrome, an inherited lymphoproliferative disorder associated with autoimmunity. N Engl J Med 1996;335:1643-9.

29 Fisher GH, Rosenberg FJ, Straus SE, et al. Dominant interfering Fas gene mutations impair apoptosis in a human autoimmune lymphoproliferative syndrome. Cell 1995;81: 935-46.

30 Siegel RM, Frederiksen JK, Zacharias D, et al. Fas pre-association required for apoptosis signaling and domipre-association required for apoptosis signaling and domi288:2354-7.

31 Boldin MP, Mett IL, Varfolomeev EE, et al. Self-association of the "death domains" of the p55 tumor necrosis factor (TNF) receptor and Fas/APO1 prompts signaling for IN and Fas/APO1 effects. J Biol Chem 1995;270:387-91

32 Chen PC, DuBois GC, Chen MJ. Mapping the domain(s) critical for the binding of human tumor necrosis factoralpha to its two receptors. J Biol Chem 1995;270:2874-8
33 Hsu KC, Chao MV. Differential expression and ligand binding properties of tumor necrosis factor receptor chimeric mutants. J Biol Chem 1993;268:16430-6.

34 Corcoran AE, Barrett K, Turner M, et al. Characterization of ligand binding by the human p 55 tumour-necrosisfactor receptor. Involvement of individual cysteine-rich repeats. Eur J Biochem 1994;223:831-40.

35 Marsters SA, Frutkin AD, Simpson NJ, Fendly BM, Ashkenazi A. Identification of cysteine-rich domains of the type 1 tumor necrosis factor receptor involved in ligand binding. J Biol Chem 1992;267:5747-50.

36 Naismith J, Devine T, Kohno T, Sprang S. Structures of the extracellular domain of the type I tumor necrosis factor receptor. Structure 1996;4:1251-62.

37 Naismith J, Devine T, Brandhuber B, Sprang S. Crystallographic evidence for dimerization of unliganded tumor necrosis factor receptor. J Biol Chem 1995;270:13303-7.

38 Tsien RY. The green fluorescent protein. Annu Rev Biochem 1998;67:509-44.

39 Tron L, Szollosi J, Damjanovich S, Helliwell SH, ArndtJovin DJ, Jovin TM. Flow cytometric measurement of fluorescence resonance energy transfer on cell surfaces. Quantitative evaluation of the transfer efficiency on a cell-by-cell basis. Biophys J 1984;45:939-46.

40 Szollosi J, Tron L, Damjanovich S, Helliwell SH, ArndtJovin D, Jovin TM. Fluorescence energy transfer measurements on cell surfaces: a critical comparison of steady-state fluorimetric and flow cytometric methods. Cytometry 1984:5:210-16

41 Chan FKM, Chun HJ, Zheng L, Siegel R, Bui K, Lenardo MJ. A domain in tumor necrosis factor receptors that mediates ligand-independent receptor assembly and signaling. Science 2000;288:2531-4.

42 Papoff G HP, Eramo A, Pagano MG, Di Leve G, Signore A, Ruberti G. Identification and characterization of a ligand-independent oligomerization domain in the extracellular region of the CD95 death receptor. J Biol Chem 1999;274:38241-50.

43 McDermott MF, Aksentijevich I, Galon J, et al. Germline mutations in the extracellular domains of the $55 \mathrm{kDa}$ TNF receptor, TNFR1, define a family of dominantly inherited autoinflammatory syndromes. Cell 1999;97:133-44.

44 Centola M AI, Kastner DL. The hereditary periodic fever syndromes: molecular analysis of a new family of inflammatory diseases. Hum Mol Genet 1998;7:1581-8. 